\title{
Recent Developments in Pickering Emulsions for Biomedical Applications
}

\author{
Clarissa L. G. Harman, ${ }^{\mathrm{a}, \mathrm{b}}$ Mehzabin A. Patel, ${ }^{\mathrm{b}}$ Stefan Guldin, ${ }^{\mathrm{b}}$ Gemma-Louise Davies ${ }^{\mathrm{a}}$ \\ a Department of Chemistry, University College London, 20 Gordon Street, London WC1H 0AJ, \\ UK \\ ${ }^{\mathrm{b}}$ Department of Chemical Engineering, University College London, Torrington Place, London \\ WC1E 7JE, UK. \\ ‘Email: gemma-Louise.davies@ucl.ac.uk
}

Keywords: Pickering emulsions, biomedicine, stimuli-responsive, therapeutic delivery

\begin{abstract}
Pickering emulsions, stabilised by organic or inorganic particles, offer long-term dispersibility of liquid droplets and resistance to coalescence. The versatility of stabilising particles and their ability to encapsulate and release cargo with high internal payload capacity makes them attractive in a wide variety of applications, ranging from catalysis to the cosmetic and food industry. While these properties make them an equally promising material platform for pharmaceutical and clinical applications, the development of Pickering emulsions for healthcare is still in its infancy. Herein, we summarise and discuss recent progress in the development of Pickering emulsions for biomedical applications, probing their design for passive diffusion-based release as well as stimuli-responsive destabilisation. We further comment on challenges and future directions of this exciting and rapidly expanding area of research.
\end{abstract}

\section{Introduction}

Emulsion-based systems have been applied for several decades in a wide variety of fields, from drug delivery and pharmaceutics to cosmetics and the food industry, thanks to their relative ease of formulation for poorly soluble drugs and other substances. Pickering emulsions, first described in the early 1900s,[1,2] are stabilised by solid particles rather than surfactants and can offer a plethora of advantages over traditional emulsions, including increased stability against solvent coalescence, lower toxicity, and added functionality, derived from the properties of the stabilising particles themselves.

Pickering emulsions stabilise the interface between two immiscible liquids by using solid nanoparticles, which reduce the interfacial energy of the system to produce a stable emulsion. Which of the two classes of emulsion that can be formed, water-in-oil (W/O) or oil-in-water $(\mathrm{O} / \mathrm{W})$, is determined by the wettability of the particles, as described by the Young equation and three-phase contact angle, $\theta$.[3] Stable Pickering emulsions are generally formed when the contact angle is close to $90^{\circ}$. Particles with moderately hydrophilic surfaces tend to form $\mathrm{O} / \mathrm{W}$ emulsions (due to $\theta<90^{\circ}$ ), whereas slightly hydrophobic particles form W/O emulsions (where $\theta>90^{\circ}$ ). If the affinity of the particles is too pronounced, the droplet will not be stabilised, resulting in a break-up of the emulsion and dispersion of the particles in the phase with the greatest wettability. The stability of Pickering emulsions is also dependent on the particle size, shape and concentration. These aspects are described thoroughly in a number of excellent review articles and therefore will not be discussed in detail in this article.[3-8] 
Pickering emulsions have been applied in a number of areas of research and industrial importance, such as food manufacturing, cosmetics, agrochemicals and therapeutic delivery. Their popularity in biomedical applications (i.e. for use in healthcare, such as therapeutics, diagnostics or imaging), in particular, has increased dramatically in recent years, thanks to their high stability, capacity for superior cargo loading compared to conventional systems, and diverse range of stabilising particles, creating a broad library of available building blocks. For biomedical and pharmaceutical application, the choice of emulsifier is critical; it must be biocompatible, non-toxic, and be able to be excreted from the body (if necessary). In this article, we will review the latest developments in the design and application of Pickering emulsions for biomedicine, with a focus on stimuli-responsive Pickering emulsions as a route to the triggered release of a payload towards advanced therapeutic delivery strategies. Within this discussion, we will describe systems which have been applied in proof of concept and in vitro assessments and emphasise areas of potential future development.

\section{Pickering Emulsions in Biomedical Applications}

The unique potential of Pickering emulsions may be most evident in applications such as delivery of therapeutics and medical imaging,[9] due to their stability, high payload capacity, and potential for bespoke modification, particularly through the exploitation of the properties of the stabilising nanoparticles themselves. Emulsifiers for biomedical Pickering emulsions are typically based on inorganic or organic particles, such as silica $\left(\mathrm{SiO}_{2}\right)$ or magnesium hydroxide,[10-13] or polymer-based systems including poly lactic-co-glycolic acid (PLGA), poly $(N$-isopropylacrylamide) (pNIPAM), polystyrene (PS), or poly(methyl methacrylate) (PMMA). [14,15] More recently, there has been a drive to implement naturally-occurring polysaccharides, for example cellulose, starch, chitosan, or alginic acid, whose biocompatibility, non-toxic properties, and biodegradability are attractive for the development of biologically relevant Pickering emulsions.[16-19]

There are a number of challenges associated with implementing Pickering emulsions in biomedical applications, typically associated with the physiological environments they are likely encounter en route to their target sites. For example, penetration of topical formulations face the difficulties of skin permeation; orally applied systems will encounter high $\mathrm{pH}$, enzyme concentrations and microbes in the oral and gastrointestinal (Gl) tract which could affect their structural integrity; and (often) large Pickering emulsion droplet sizes may pose problematic in intravenous delivery. These obstacles must be considered at the design stage of Pickering emulsions to ensure they remain relevant and robust for potential clinical application.

A related challenge lies in preventing premature release of cargo from Pickering emulsion formulations. Some stabilising particles themselves have been directly linked to early release of cargo, often due to their porosity and structure.[20-23] Small (bio)molecules such as active pharmaceutical ingredients (APIs) can become entangled within the polymer chains (of polymer-based Pickering emulsions or microcapsules) or remain adsorbed on external emulsion surfaces, resulting in early 'burst' release.[20,22,23] Such issues can, of course, be avoided through judicious washing, or modification of components. Strategies such as increasing the shell thickness, cross-linking or solidification of the Pickering emulsion surfaces, or using a combination of particles for droplet stabilisation, can prevent untimely 
cargo leakage.[21,23,24] There are, therefore, a number of considerations in the design of a successful therapeutic Pickering emulsion formulation.

\subsection{Therapeutic Delivery using Pickering Emulsions}

For the delivery of therapeutics, the emulsion stability is crucial with respect to the shelf life of the product and avoidance of systemic exposure in order to minimise side-effects. As a result, the majority of Pickering emulsions rely on therapeutic diffusion based delivery or distribution following emulsion destabilisation through degradation, for example in the low $\mathrm{pH}$ of the stomach. There are a number of routes for therapeutic delivery which have been explored and within these, significant modification of design principles must be used in order to develop effective delivery systems.

\subsubsection{Topical Application}

The topical administration of therapeutics involves local application to external surfaces such as skin or mucosa. Topical formulations are traditionally gels, creams, ointments, foams, aerosols, or lotions and their appeal stems from their ease of application by the end user (rather than requiring clinician application), local therapeutic effect and minimal adverse systemic side effects. For such agents to be successful, they must penetrate the epidermis, which can act as a barrier to the delivery of many impermeable therapeutics, and provide effective and sustained drug release. The adhesive ability and degree of penetration of a topically applied therapeutic is dependent on the product formulation itself. This remains the largest challenge in the development of dermally-applied Pickering emulsion systems, requiring significant development of the carrier system for effective function. Within this, careful consideration must also be given to ensuring that any formulation does not cause side effects, such as skin irritation or dermatitis. Pickering emulsions offer an interesting route to improving dermal drug permeation, since their formulation allows the incorporation of penetration-enhancing molecules, alongside their stability and high payload capacity.[6]

Pioneering work by Chevalier and co-workers demonstrated the first example of W/O silica particle-based Pickering emulsions for the transdermal delivery of caffeine.[10] Compared to a traditional classical surfactant-stabilised emulsion, this Pickering emulsion showed a 3-fold higher transdermal permeation rate. This was related to improved adhesion of the Pickering emulsions to the skin surface, as well as deep skin penetration of the stabilising silica nanoparticles, leading to enhanced drug release. Subsequent work on O/W Pickering emulsions demonstrated their capability for targeting different, in particular deeper, skin layers due to their slow release capacity.[11] More recent work has shown that the choice of formulation emulsifier and oil can affect the permeability of the Pickering emulsions, its depth of penetration within the skin, and accumulation of particles, therefore affecting drug efficacy.[18-20] For example, using oils such as glycerol and evening primrose as permeation promoters, Wang et al. observed increased therapeutic delivery which they related to the structural distortion of the stratum corneum skin layer by the oils.[20] Hu et al. further validated this idea, establishing that the structure of the oil was critical not only for the stability of the Pickering emulsion, but also for controlling depth of penetration and accumulation within skin.[18] Oils containing ring-structures allowed the highest permeation through the skin, with linear chain oils showing the highest skin retention. This again indicates that careful choice of Pickering emulsion formulations allow regulation of the skin target site, an important consideration in topical applications. 
The topical application of Pickering emulsions can be beneficial not only for direct therapeutic delivery, but also for skin decontamination, as has recently been demonstrated by Salerno et al., who developed Pickering emulsions for the removal of the chemical warfare agent VX.[12] In this case, the Pickering emulsion containing a warfare scavenging agent exhibited higher efficiency in the removal of the toxin than the scavenging agent alone. This was attributed to the strong adhesion of the emulsifiers to the skin surface, which aided in transfer of the chemical warfare agent from the skin to the oil phase of the Pickering emulsion (Figure 1a). Other recent work has used chitosan based Pickering emulsions as a method to enhance wound healing, where the synergistic effect of the cargo therapeutic and other components of the Pickering emulsion demonstrated improved functioning compared to the drug alone.[16] Another innovative topical application of Pickering emulsions is for sunscreens,[25,26] where the emulsifying particles can be physical UV filters in combination with an encapsulated active agent, such as melatonin.[27] Such formulations can present a stable and effective sunscreen with the added benefit of protection against oxidative stress, thanks to melatonin's free radical scavenger and antioxidant activity.[27] Silica particle-stabilised emulsions containing a dissolved UV filter have also recently been demonstrated to produce effective sunscreen films due to evaporation-induced effects.[26] Their unique combination of volatile and involatile components allows the UV filter to remain soluble throughout evaporation, maintaining excellent sun protection. These emulsion films offer advantages over solution sunscreen films, thanks to reduced film shrinking, which circumvents potential loss of sun protection.

These diverse applications of Pickering emulsions showcase their varied potential applications in topical biomedicine, with clear synergistic enhancements compared to traditional topical formulations. Increased activity and efficacy of the Pickering emulsions in these applications is linked directly to the design of the particulate emulsifier agents. These particles often demonstrate enhanced adhesion, resulting in increased skin adhesion and hence improved delivery efficacy, avoiding the requirement for chemical penetration enhancers. Such increased delivery efficiency, coupled with long shelf life and recent advances in the use of biocompatible and biodegradable emulsifiers,[14] exemplify the unique benefits of Pickeringbased systems over traditional topical designs and offer an exciting new direction in topical formulations.

\subsubsection{Oral Application}

Orally applied therapeutics offer obvious opportunities to treat internal organs, in particular in the GI tract, and their ease of application makes them highly desirable. Oral application does, however, pose unique challenges due to the systemic distribution of a drug after entering the GI tract and subsequently the blood stream, which can result in unwanted interactions with receptors, tissues and organs, causing undesirable, and potentially dangerous side effects. Unpredictable adsorption from uncontrollable degradation by oral enzymes, microbe environments, and/or stomach acid additionally means that medicines often require a structural barrier or preservative such as an enteric coating, a challenge which is also pertinent to the use of Pickering emulsions for oral delivery routes. On the other hand, exploitation of the $\mathrm{pH}$ and enzymatic environments that a formulation will encounter during ingestion can facilitate the development of formulations whose release is enhanced by, or even relies on, these environments, for example, initiating release of an active pharmaceutical specifically in the oral or gastro-cavity. 
Pickering emulsions designed on this basis promise advantages over traditional (enteric) coating methods, based on their high surface areas and bespoke surface functionality. Cossu et al., for example, developed starch-based Pickering emulsion formulations for the treatment of oral infections of $C$. albicans with sensitivity to the oral enzyme $\alpha$-amylase.[17] These O/W Pickering emulsions demulsified in response to the addition of $\alpha$-amylase due to the enzymatic digestion of starch, initiating the controlled-release of the encapsulated active anti-fungal therapeutics thymol and amphotericin B. In a related effort, Sy et al. exploited the acidity of the $\mathrm{GI}$ tract for controlled destabilisation and cargo release of O/W Pickering emulsions based on the dissolution of the emulsifying $\mathrm{Mg}(\mathrm{OH})_{2}$ particles at low $\mathrm{pH}$.[28]

These tactics rely on the response of the emulsifier to the external environment to destabilise and release the therapeutic encapsulated in the Pickering emulsion. An alternative route has recently been described which exploits the therapeutic agent itself as the emulsifier.[29] The drug silybin, used to treat liver damage, has poor water solubility and bioavailability, however, silybin nanocrystals, which adsorb at the oil-water interface, have been demonstrated to behave as both an emulsion stabiliser and an active therapeutic. High drug release (compared to silybin alone) occurs due to the partial dissolution of the drug into the oil phase of the Pickering emulsion, leading to ready release and high efficacy. This is attributed to the formation of lipid-like drug solutions which interact with endogenous solubilising species (for example phospholipids, cholesterol or bile salts) and promote transfer into the aqueous phase for improved bioavailability. Careful choice of the stabilising particle therefore has the opportunity to transform the efficacy of Pickering emulsions.

\subsubsection{Parenteral Application}

Pickering emulsions further offer potential for parenteral applications, such as intramuscular, intravenous, and subcutaneous routes. As with oral applications, parenteral administration is systemic, therefore, enhancing the targeting ability of a formulation is beneficial to reduce undesirable side effects. Exploiting targeting agents has recently shown enhanced efficacy of W/O Pickering emulsions of oxaliplatin, a liver tumour chemotherapeutic, and Lipiodol stabilised with biodegradable PLGA particles, designed for intravenous delivery.[30] Key to this work was the targeting ability of the Lipiodol, an oil-based radio-opaque contrast agent which shows preferential tumour uptake. In comparison to conventional W/O emulsions, sustained release of the chemotherapeutic was observed in combination with reduced systemic exposure. Due to the reduced toxicity to non-diseased organs, these findings could lead to an extension of the therapeutic window, i.e. the ability to increase chemotherapy doses without the usual associated systemic side effects, which would be of vast importance in future patient therapies.

A challenge associated with intravenous formulations is designing their controlled, sustained release, which can significantly lower systemic exposure to non-target organs, and aids in significant release of the therapeutic at the target site. Neufeld and co-workers have been working on such slow-release systems for a number of years,[31,32] most recently preparing W/O Pickering emulsions stabilised by glycerol monostearate for the delivery of an anti-cancer therapeutic oseltamivir phosphate which targets mammalian neuraminidase 1 involved in multistage tumorigenesis in a number of cancer types.[33] Efficient encapsulation of the active drug into the Pickering emulsions provided a stable formulation with slow-release properties, however the excellent stability led overall to only a low fraction of the active compound being released. The addition of a surfactant to the system, in this case sorbitan monooleate, 
facilitated increased therapeutic release as a result of surfactant adsorption onto emulsifier particle surfaces. This altered their wetting behaviour and stimulated emulsion destabilisation. Sustained drug release was observed over a 30-day period, increasing the therapeutic effect against pancreatic cancer cells. Further work in this area to enable tight control over the duration of the therapeutic release could offer a unique opportunity for future patient treatments.

In an alternative, but related subcutaneous delivery application, Pickering emulsions have very recently been explored as adjuvants towards new vaccine formulations.[34] PLGA-stabilised Pickering emulsions with immobilised antigens produced by Xia et al. provided the forcedependent deformation and mobility necessary to enable multivalent interactions with antigenpresenting cells, enhancing cellular internalisation of the emulsion droplets (Figure 1b). These properties provided significant advantages over conventional emulsions, with enhanced antigen binding, uptake and activation making this a potent adjuvant for vaccine delivery with enormous potential. Similar sustained delivery of vaccine antigens has been observed using polymeric bioresorbable amphiphiles as emulsifying agents for W/O systems, enhancing vaccine efficacy.[35]

Whilst parenteral application of Pickering emulsions clearly shows strong promise, enabling targeting and sustained release, a major challenge is the physical construct of the formulation itself. Pickering emulsion droplets can be relatively large (up to several microns in size), which may prove problematic for effective delivery, and evasion of the mononuclear phagocytic system (MPS).[36] The subsequent biodistribution of such emulsions, as well as their breakdown products (i.e. stabilising particles following demulsification/dissolution) also require careful consideration. It has been noted that the shape and size of injected particles can have an effect on their biodistribution and accumulation within organs.[37] This is rarely mentioned in articles describing Pickering emulsions for therapeutic delivery in the literature, but it is a sizeable problem for translation to the clinic, and should be afforded more consideration in future investigations.

\subsection{Pickering Emulsions in Biosensing and Bioseparation}

Pickering emulsions can additionally be used for biorecognition and bioseparation by harnessing molecular imprinting technology. This technique traditionally uses polymeric matrices to design materials (molecularly imprinted polymers, MIP) capable of molecular recognition with strong binding affinities and high selectivity towards a (bio)molecule of interest, mimicking natural recognition systems such as antibodies and biological receptors.[38,39] Whilst already an established field, there are some reservations surrounding MIP generated though polymerisation, as the generated polymers often have limited control over the chain length, resulting in irregular morphologies or shapes.[40] Pickering emulsion polymerisation, on the other hand, where polymers are formed within the emulsion internal phase, typically generates polymers with controlled and well-defined lengths thanks to their regulation by the emulsion droplet size.[40] A variety of different biomolecules and chemicals have been imprinted in this way, facilitating targeting of proteins,[41] steroids,[42] bacteria,[43] and APIs.[40] A recent interesting application of Pickering emulsion enabled MIP is shown in the work of Hajizadeh et al., who developed MIP immobilised in cyrogels for the capture and purification of haemoglobin $\mathrm{Hb}$ protein from cell homogenate suspension and non-purified red blood cells lysate.[24] This work demonstrated clear advantages over traditional immobilisation strategies, with the Pickering emulsion-formed MIP exhibiting high binding 
capacity and enhanced selectivity towards $\mathrm{Hb}$ proteins as a result of excellent accessibility of the active MIP groups. This bioseparation behaviour of MIPs formed using Pickering emulsions has been demonstrated in a number of other works, demonstrating the utility of Pickering emulsions in diverse biomedical applications.[21,44]
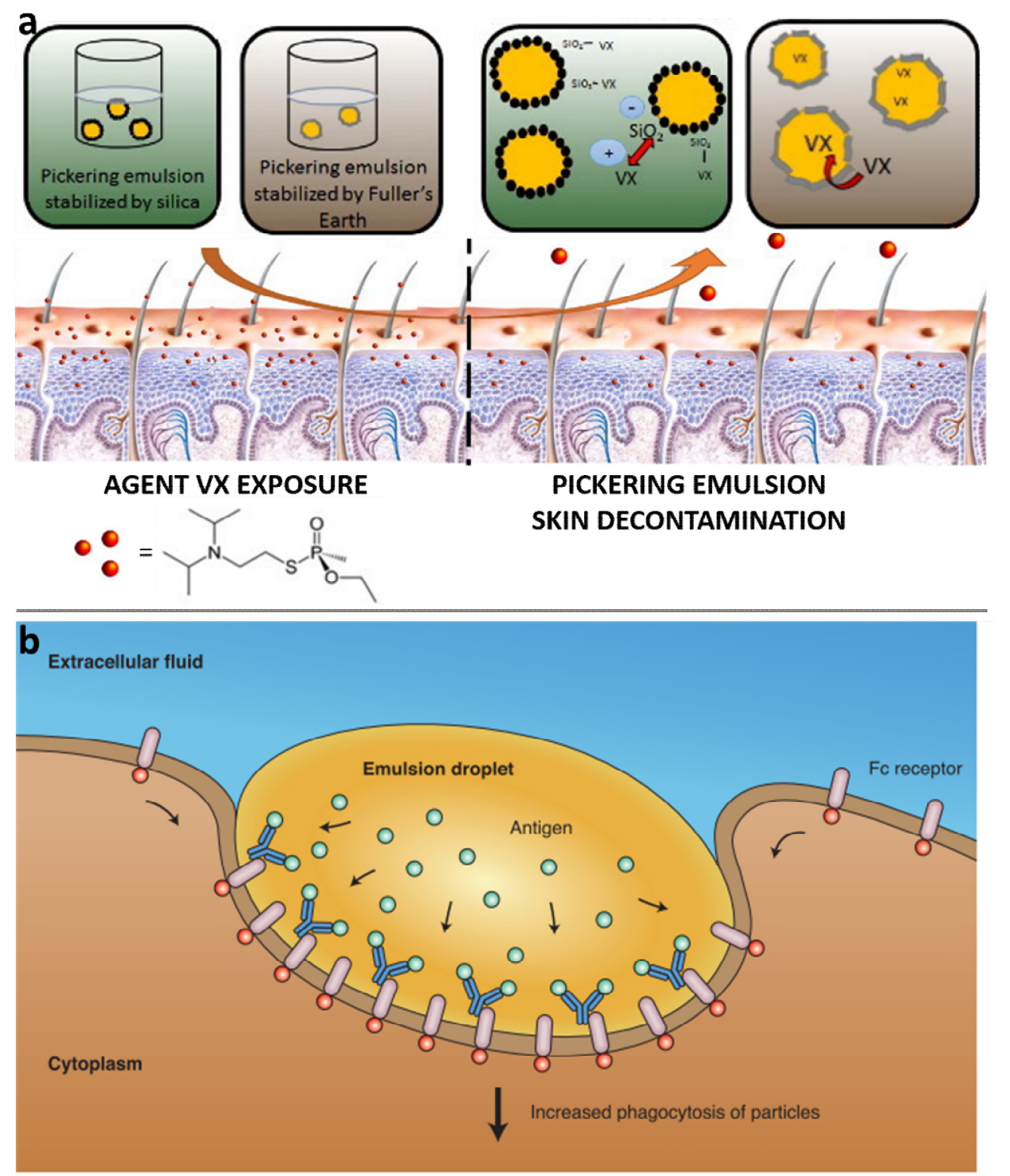

Figure 1. Examples of the use of Pickering emulsions in different therapeutic delivery approaches: a) Schematic showing the topical application of Pickering emulsions for skin decontamination with enhanced scavenging and removal of a chemical warfare agent due to migration into the oil phase and electrostatic interactions; image adapted, with permission, from Elsevier copyright 2016.[12] b) Schematic showing a Pickering emulsion adjuvant system with enhanced cell delivery of an antigen (green sphere). Increased contact of the emulsion droplet with the cell surface, due to shape deformation, facilitates antigen-antibody (blue " $Y$ " symbol) binding at the interface (Fc receptor-mediated process), boosting cellular internalisation of the emulsion droplets; image adapted with permission from Nature Publishing Group copyright 2018.[34,45] 


\section{Stimuli-responsive Pickering Emulsions}

Conceptually, it is evident that colloidal stability and stimuli-induced demulsification are key to the controlled release of cargo. All of the Pickering emulsion based delivery systems described thus far have been relatively stable systems, which rely on slow diffusion-based release or known exposure to specific environments (such as low pH stomach acid or enzymes) to allow destruction and hence therapeutic 'escape' from the stable emulsions. However, the concept of a Pickering emulsion that remains stable until the application of an external trigger, offers opportunities to control therapeutic delivery directly at the disease site.[46] This could increase efficacy, reduce overall required doses and potentially diminish side effects - of enormous benefit to the patient.

Tissues, organs and cells are precision machines, and small changes in their microenvironment and behaviour can be indicative of the presence of diseased tissues and tumours. For example, certain enzymes are well-known to be linked with specific disease pathologies, including stroke, cardiovascular or neurodegenerative inflammatory responses and cerebral ischemia;[47] metal ions play vital roles in a number of important signalling pathways in the body, with changes in concentrations being indicative of diseases such as Alzheimer's (increased $\mathrm{Zn}^{2+}$ levels);[48] pH can also be monitored in the detection of ischemia or metabolic disorders.[49] The presence or variation of such species has previously been exploited in order to produce useful biomedical systems, such as therapeutic delivery from nanoparticles as well as diagnostic imaging agents,[46,50] however Pickering emulsions possessing this responsive capability have been less widely probed.

The design of Pickering emulsions which can be manipulated by the use of external stimuli has received increasing attention in recent years, with examples of droplet destabilisation in response to $\mathrm{pH}$, salt concentrations, chemical or biological entities, temperature, light, shear, microfluidic collision, electric and magnetic stimuli.[9,51-57] In general terms, a transformation in the emulsifier upon exposure to a stimulus can result in a change in the emulsion stability. This transformation may distort the size of the particle, forcing it to swell or shrink; or initiate phase inversion due to a change in emulsifier wettability; or cause complete demulsification and disassembly of the emulsion (which can be reversible or irreversible). Stimuli-responsive Pickering emulsions have been reported for various applications in product recovery, oil recovery, and catalysis,[6] however their potential in biomedical applications has only started to emerge in the past decade.[58]

\section{1 pH Responsive Pickering Emulsions}

Variation of $\mathrm{pH}$ in different regions of the body is well known and can be a useful indicator of the presence of disease. Malignant tumours present with $\mathrm{pH}$ ranging from 6.8 to 7.2 , and the presence of hypoxia, tumour growth and metastases are generally indicated by regions of greater acidity than blood and healthy tissue.[59] $\mathrm{pH}$ changes can therefore prove useful for triggered therapeutic release directly at a site of disease and is one of the most popularly probed release mechanisms in stimuli-responsive Pickering emulsions.

For the purposes of drug delivery via this stimulus, it is important to ensure the stability of the Pickering emulsion at physiological $\mathrm{pH}$, with the cargo stably encapsulated until release at the desired $\mathrm{pH}$ trigger location.[46] Traditionally, highly charged small molecules or surfactants are employed to stabilise the surface of the emulsifying nanoparticle in a physiologically relevant $\mathrm{pH}$ range through electrostatic interactions.[60-63] Variation in $\mathrm{pH}$ subsequently 
breaks these electrostatic interactions through protonation/deprotonation, leading to demulsification due to changes in particle wettability and subsequent cargo release. The same principles of triggered demulsification due to changes in electrostatic stabilisation and wettability can be exploited with the use of polymer-grafted inorganic nanoparticle emulsifiers.[64,65] Phase inversion can be observed with carefully-designed long-chain polymeric emulsifiers, such as polyurethanes or amphiphilic Janus particles. $[66,67]$ In all these systems, care must be taken to ensure the species employed are biocompatible, and do not cause irritation, or unsought responses.

Biocompatible materials including polysaccharides like chitosan,[68,69] soy peptides,[70] lignin[71] and alginic acid[72,73] show excellent potential as emulsifiers and emulsifiermodification agents in stimuli-responsive Pickering emulsions. This is due to the abundance of $\mathrm{pH}$-responsive functional groups in their structures, such as amines, hydroxyls, and carbonyls, which can cross-link and dissociate or decompose accordingly to stabilise or destabilise the emulsion systems.[74] The future of $\mathrm{pH}$-responsive systems may lie within combining natural polymers and inorganic materials to form so-called double Pickering emulsion systems comprising multiple components, each capable of tailored and controllable release of different therapeutics systematically. Recent efforts towards this by Guo et al., have used graphene oxide (GO), polylactic acid (PLA), and hydroxyapatite (HA) to construct core@shell@shell GO@PLA@HA colloidosomes as a multistage drug-release system. This multi-component system was prepared via the double Pickering emulsion method and microcapsules were formed following volatile solvent escape from the initial droplets. The materials within the composites were chosen for their biocompatibility and differing surface chemistry which permits drugs of differing hydrophobicity (rose Bengal, coumarin and 5fluorouracil) to be encapsulated (Figure 2a).[51] The pH-sensitivity of the HA outer shell, which undergoes acid degradation, enabled controlled drug release of this multicomponent system, with efficient drug release at $\mathrm{pH}$ 5.0. The multi-layer system additionally facilitated staged and sustained release of the different drug components, as the emulsion degraded. This example of a solidification of a double Pickering emulsion system shows exceptional promise and inspiration can be taken for the next generation of drug-delivery carriers, where multi-drug systems can increase therapeutic efficacy, in particular for efficient tumour therapy.

One of the most important considerations in the development of $\mathrm{pH}$-responsive Pickering emulsions is the sensitivity and $\mathrm{pH}$ range of the release mechanism. The $\mathrm{pH}$ environment within the body that a formulation may encounter varies dramatically between organs, blood, tumour or infection sites. This necessitates that designed formulations remain stable across a wide $\mathrm{pH}$ range and only exhibit a narrow well-defined $\mathrm{pH}$-responsive window, such that their triggered release occurs only at the desired site. While many of the systems described herein demonstrate stability over a relatively wide $\mathrm{pH}$ range, and release at a specific $\mathrm{pH}$ range, it remains unclear how robust and narrow the $\mathrm{pH}$ window remains under physiological conditions. More work is required before any translation to in vivo studies could be considered.

\subsection{Photoresponsive Pickering Emulsions}

Premature release can be a major issue associated with exploiting internal biological markers as a trigger for Pickering emulsion destabilisation and therapeutic release. A great deal of research has hence focussed on the use of stimuli which can be controlled ex situ by an end user or clinician.[75] Light-based clinical treatments are already popular, for example the use of near-infrared (NIR) radiation in photothermal therapy (PTT) for the treatment of cancer[76] 
and photodynamic therapy (PDT) in the treatment of skin cancer and dermatological issues such as acne, cutaneous infections, or inflammatory disorders.[77] The benefit of these stem for the precise placement of the light source, minimising exposure and hence side effects, although poor tissue penetration means that the utility of such treatments can be limited.

Light-responsive Pickering emulsions for biomedical applications are a steadily expanding area of research, although only a handful have been described in recent years. Similarly to the $\mathrm{pH}$-responsive systems, their mechanism of release follows light-irradiation triggered changes in emulsifier particle wettability, leading to Pickering emulsion inversion[78-80] or coalescence. $[52,81]$ Changes in wettability are again proffered by the surface modification of the stabilising particle. The optically sensitive molecule spiropyran (which undergoes a conformational change with light irradiation), when associated with silica particles, for example, produces an amphiphilic system which switches from being hydrophobic to hydrophilic upon UV illumination, resulting in emulsion inversion.[78,79] This approach has been demonstrated to be particularly useful for biocatalysis, where inversion aids product recovery and emulsifier recycling.[79] Stenhouse adducts, an alternative class of photoactive donor-acceptor molecules, have very recently been examined as an alternative emulsifier modification route to visible light triggered inversions of Pickering emulsions.[80] Their photoactivated transition between hydrophobic to zwitterionic species efficiently and quickly alters particle wettability and is the first example exploiting these interesting compounds for cargo release, as demonstrated using model dye compounds.

Photocatalytically active materials, such as $\mathrm{TiO}_{2}$, have clear advantages as Pickering emulsion stabilisers for the production of light-responsive systems. They rely on the formation of surface defects upon light illumination; the production of oxygen vacancies at bridging sites results in the conversion of $\mathrm{Ti}^{4+}$ to $\mathrm{Ti}^{3+}$ species which favour dissociative water adsorption, producing the necessary change in wettability to cause emulsion destabilisation.[52,81] Importantly, this triggered demulsification can be carried out using UV, NIR or visible light, when $\mathrm{TiO}_{2}$ or $\mathrm{N}$-doped $\mathrm{TiO}_{2}$ nanocomposites are employed as emulsion stabilisers (Figure 2b). $[52,81]$ Bai et al. have demonstrated this mechanism for the triggered release of an encapsulated API, astragalus polysaccharides.[52]

Despite the relatively few examples of light-actuated Pickering emulsions for biomedical applications, the potential of these systems is clear. When designing light-responsive Pickering emulsions, consideration must be taken when choosing a light source; UV light, for example, is a major contributor to skin cancer development.[82] NIR, on the other hand, has deeper biological tissue penetration and low scattering, making it a useful non-invasive clinical tool. In addition, the impact of reactive by-products, such as reactive oxygen species, on nontargeted cells must be minimal or non-existent to prevent damage to healthy cells.

\subsection{Thermoresponsive Pickering Emulsions}

Another approach to remote-controlled Pickering emulsion cargo release is the use of temperature. Thermal ablation is a common clinical treatment, for example in the hyperthermic treatment of cancer. Heat application is therefore readily available and can have slightly deeper tissue penetration than light irradiation. The exploitation of varying temperature within the body may be an alternative route of triggering therapeutic release; due to their increased metabolism, it is well-known that cancerous tissue locally presents with slightly elevated temperatures $\left(40-42^{\circ} \mathrm{C}\right)$ compared to healthy tissue $\left(37^{\circ} \mathrm{C}\right)$. This small thermal window has 
been explored in other release and diagnostic systems and may be exploited for emulsion destabilisation.[83]

In order to achieve thermally sensitive Pickering emulsions, control over stabilising particle wettability can be harnessed through the exploitation of thermoresponsive polymers. Their functionality is typically based on a conformational transformation with changing temperature. When utilised in Pickering emulsions, this change in geometry can cause destabilisation or increased permeability, causing cargo release.[84] Poly( $N$-isopropylacrylamide), pNIPAM, is one of the most extensively used thermoresponsive polymers for biomedical applications as its conformational transition temperature is close to biological temperatures $\left(32^{\circ} \mathrm{C}\right)$ and it can be easily modified. Within Pickering emulsion research, pNIPAM can be used independently as the emulsifier[85] or grafted onto other materials to achieve a responsive stabilising agent.[22,84,86-88] Below the polymer transition temperature, in its hydrophilic state, Pickering emulsions remain stable, however when raised above this temperature, their hydrophobic transformation alters particle wettability and disintegrates the emulsion. This approach holds advantages due to its reversibility and the narrow temperature range and timeframe of transition.

Thermoresponsive materials are not only limited to drug delivery. Recent work by Chen et al., developed similar systems for biosensing/bioimaging applications.[9] In this study, carbon dots (CD) were incorporated into pNIPAM and grafted onto cellulose acetate nanocrystals (CA). It was observed that the formed composites had temperature dependent 'on/off' fluorescence switching behaviour. Below the transition temperature, fluorescence was turned on, however above it, fluorescence was turned off (Figure 2c).

Alternatively, thermally-responsive Pickering emulsions can be achieved through emulsifier particle grafting with a stabilising surfactant whose weak interaction with the core stabilising particle can be broken through a change in temperature. Binks and co-workers recently demonstrated this approach using silica particles stabilised with the non-ionic surfactant alkyl polyoxyethylene monododecyl ether.[89] Hydrogen bonding between the oxygen atoms of the polyoxyethylene headgroup and nanoparticle surface silanol groups facilitated adsorption and stabilisation of the emulsion at low temperatures $\left(25^{\circ} \mathrm{C}\right)$. Loss of hydrogen bonding and hence emulsion destabilisation occurred when the temperature was raised $\left(45^{\circ} \mathrm{C}\right)$. The drawback to this approach was the time taken for demulsification to occur (up to $45 \mathrm{mins}$ ). Although such behaviour this may reduce efficacy of stimuli-induced drug delivery at a target site, it may hold advantages in the slow release of therapeutic, of benefit for bolus-delivery.

While the potential for thermoresponsive systems is clear, issues could arise in its translation to clinic; the window in temperature difference between healthy and cancerous cells is narrow and therefore careful and precise design of the Pickering emulsion is critical to prevent destruction or contamination of healthy cells. 

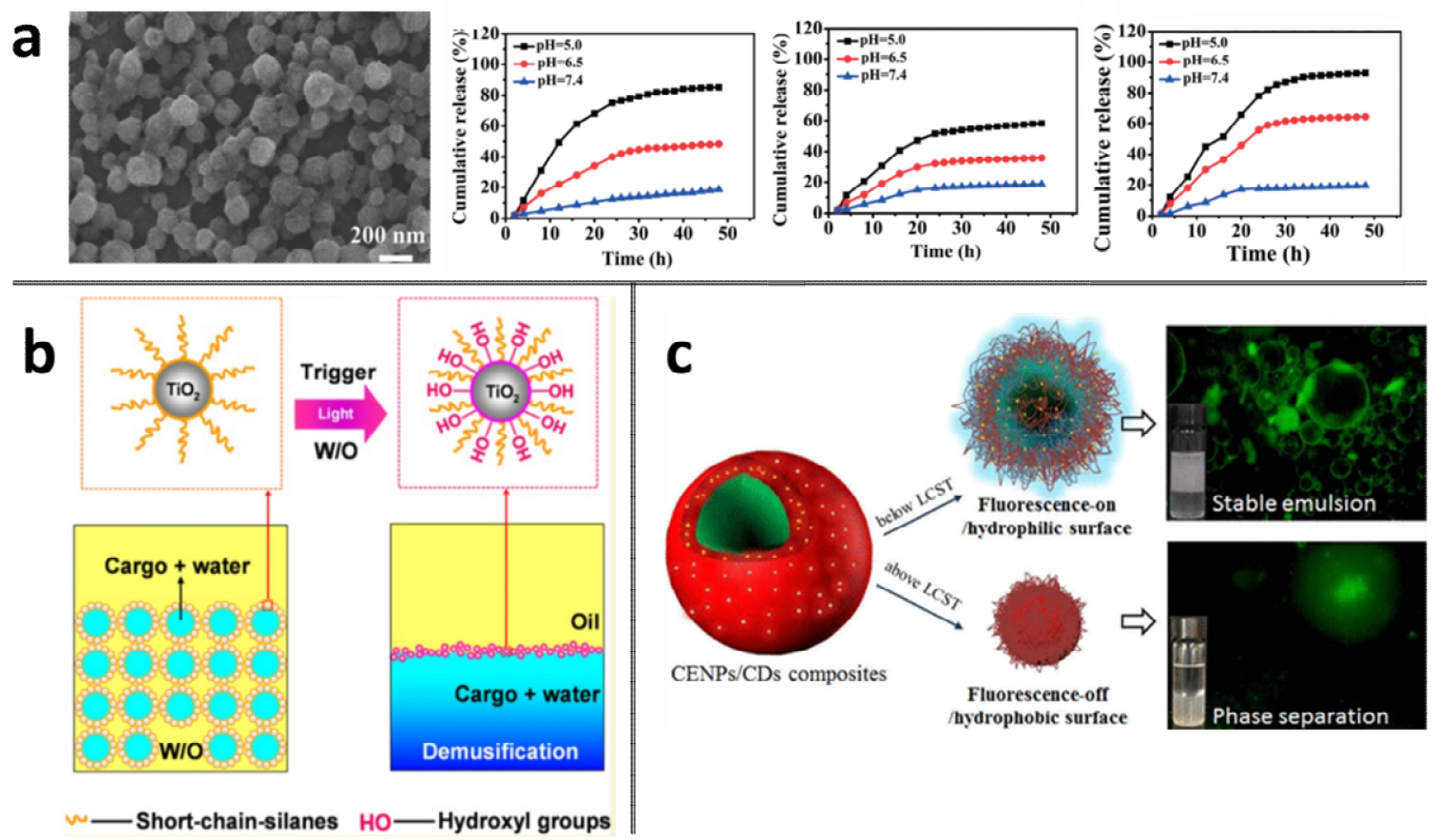

Figure 2. Examples of stimuli responsive Pickering emulsions. a) Left to right: SEM image of pH-responsive core@shell@shell GO@PLA@HA composite microcapsules formed from W/O/W Pickering emulsions and cumulative release profiles of rose Bengal, coumarin and 5fluorouracil, respectively, from the composites containing each of the 3 drugs. Different rates of drug release were observed at different $\mathrm{pHs}$, with staged and sustained release from the multi-component system; image adapted, with permission, from Wiley copyright 2017.[51] b) Light responsive destabilisation of silane-modified $\mathrm{TiO}_{2}$ Pickering emulsions, which occurs following light irradiation as a result of increased dissociative water adsorption onto $\mathrm{TiO}_{2}$ particle surfaces, changing wettability and destroying the stable Pickering emulsion. The use of $\mathrm{N}$-doped $\mathrm{TiO}_{2}$ particles extends light response to the visible range; image adapted, with permission, from the American Chemical Society copyright 2016.[52] c) Temperatureresponsive core/shell nanospheres of cellulose acetate encapsulated by $\operatorname{poly}(\mathrm{N}$ isopropylacrylamide) (pNIPAM) with incorporated fluorescent carbon dots (CDs). Reversible emulsion breakage can be triggered through thermal changes, exploiting the conformational transition temperature of the pNIPAM layer. These composites exhibited fluorescence 'on/off' switching behaviour in response to thermal triggers and changes in the emulsion stability; image adapted, with permission from the American Chemical Society copyright 2018.[9]

\subsection{Magnetically Responsive Pickering Emulsions}

The use of magnetic materials in biomedicine has been popular for several decades, with applications as contrast agents for medical imaging as well as therapeutic delivery and bioseparation thanks to their attractive magnetic properties.[90,91] Within Pickering emulsions, inorganic magnetic nanoparticles have been explored as emulsifier agents, most commonly with the aim of producing systems whose direction and motion can be controlled using an externally applied magnetic field whilst maintaining the integrity of the Pickering emulsion.[92-94] Such species are promising for magnetic targeting to enhance drug release at a specific site whilst minimising systemic exposure,[93] though little work has shown practical demonstrations of this behaviour in vitro or in vivo. An interesting application of magnetic particle-stabilised Pickering emulsions is for scavenging and removal of unwanted 
species. Lee and co-workers showed the absorbance of a model dye compound into the aqueous phase of a magnetic Pickering emulsion, which can then be magnetically removed and recycled.[94]

The magnetic properties of the particles within magnetic Pickering emulsions may also be exploited for triggered emulsion destabilisation and cargo release. This can be through the application of an alternating magnetic field which results in heating of the particles in a manner comparable to that used in hyperthermic treatments. This approach, usually combined with a thermoresponsive polymer, relies on destabilisation of the Pickering emulsion by wettability changes dictated by the polymer's thermal transition, as described in section 3.3.[95] In fact, there are few examples of the direct effect of the application of an external magnetic field to a magnetic particle stabilised Pickering emulsion.[96] Of particular relevance to biomedicine is work by Fuller and co-workers, who observed reversible magnetically-triggered phase separation of a magnetic particle stabilised Pickering emulsion leading to emulsion destabilisation.[97] In their highly-tuned systems, the force of the movement of magnetic particles towards the magnetic field caused pressure which forced the films between droplets to thin and destabilise, removing the particles from the droplet entirely. Without a doubt, the ability to break an emulsion reproducibly using an externally applied magnetic field is extremely promising from a biomedical perspective, due to the low cost of the required magnetic systems, the depth of penetration and the fast triggering of the demulsification. This is an area which deserves further attention.

\subsection{Multi-stimuli Responsive Pickering Emulsions}

The future of stimuli-responsive Pickering emulsions for biomedical applications may lie within dual or multi-stimuli responsive systems, enabling controlled and staged release of therapeutics, or multi-stage biosensing, providing greater control, enhancing efficiency and selectivity. Combination (or multi-drug) anti-cancer therapy, for example, has become popular as a route to overcoming drug resistance, with ideal "cocktail therapies" capable of controlled release of each drug individually to maximise synergistic effects.[98] Acidic and mildly hyperthermic microenvironments of cancerous tissues could also be exploited as dual triggers for the targeted delivery of therapeutics directly at a site of disease. There has hence been a surge of research in the area of multi-responsive Pickering emulsions in the last 5 years for various applications, including industrially relevant processes such as catalysis and sensing.[99,100] Though promising, only a handful are directly applicable to or have been demonstrated for biomedical applications. [53,101]

A range of stimulus combinations have been investigated, such as $\mathrm{CO}_{2}$ and light,[99] $\mathrm{pH}$ and light,[100] $\mathrm{CO}_{2}$ and redox environments,[102] temperature and ionic strength,[103] pH and temperature,[104-106] $\mathrm{pH}$ and magnetic fields,[101,107-109] and magnetic fields and temperature.[23,110] Many systems employ emulsifiers composed of a single amphiphilic polymer with multi-functionality or co-polymer systems bearing different stimuli-sensitive groups (often based on poly( $N$-isopropylacrylamide) or poly(methyl methacrylate) species), either as a polymeric emulsifier or via modification of an inorganic particle.[23,99,100,105,106] These polymers undergo a conformational change in response to different stimuli, usually based on a conversion from hydrophilic to hydrophobic state leading to a change in wettability and demulsification[105] or phase inversion,[99] or alternatively result in swelling/shrinking of the emulsifying particles,[100] leading to reduced emulsion stability. A noteworthy example is the use of amphiphilic double dynamers which exploit both supramolecular and intramolecular 
dynamics to provide dual $\mathrm{pH}$ and temperature sensitivity resulting from morphological transitions leading to emulsion destabilisation (Figure 3a).[104]

Most studies combining magnetic responsiveness allow sensitivity to some primary stimulus (e.g. $\mathrm{pH}$ ), with the magnetic component additionally facilitating directional motion.[23,107,108] The group of Meng and co-workers have exploited a magnetic field to trigger emulsion coalescence and a chemical reaction only in the presence of UV light in a truly multiresponsive manner (Figure 3b).[109] Pickering emulsions formed using a dual-emulsifier system of magnetic and titania particles aggregated near to an applied magnetic field. Upon UV irradiation, water surface adsorption onto titania particles (following the mechanism described in section 3.3)[52,81] led to a change in wettability and coalescence between the aggregated neighbouring droplets. This facile approach would facilitate a reaction between two cargoes, allowing difficult, highly reactive or toxic reactions to take place in a controlled manner at a site of interest. An alternative use of magnetic particles is to harness their magnetic heating behaviour through the application of an alternating magnetic field. This was originally used over a decade ago to provide (magnetic) directionality as well as hyperthermiagenerated thermal demulsification,[110] but more recently has demonstrated enhanced $\mathrm{pH}$ triggered Pickering emulsion destabilisation.[101] In the presence of an alternating magnetic field, destabilisation of magnetic nanocellulose stabilised Pickering emulsions occurred at alkaline $\mathrm{pHs}$, due to the dual effect of droplet deformation upon magnetisation of the emulsifier, as well as the local field-induced thermal heating effect. This caused increased particle wettability due to increased surface adsorption of water, behaviour not observed in the absence of a field.

Another interesting recent example of a multi-responsive system by Hong et al. describes the use of a OM Pickering emulsion to form microcapsules with Au nanoparticles mutually connected by $\alpha$-synuclein proteins, through the solidification of the Pickering emulsion.[53] These systems, designed to be sensitive to disease-specific physiological properties, demonstrated protease-dependent release. As a result of disruption of the $\alpha$-synuclein- $\alpha$ synuclein interaction, controlled cargo release was facilitated, which, in combination with light sensitivity, provided photoelectronic and photothermal effects such as localised heating of the Au-based emulsifier particles. These solid colloidosomes could hold exceptional promise towards targeted cargo-carrying Pickering emulsions capable of precise pathological site recognition, of use for future sensor and therapeutic applications. 

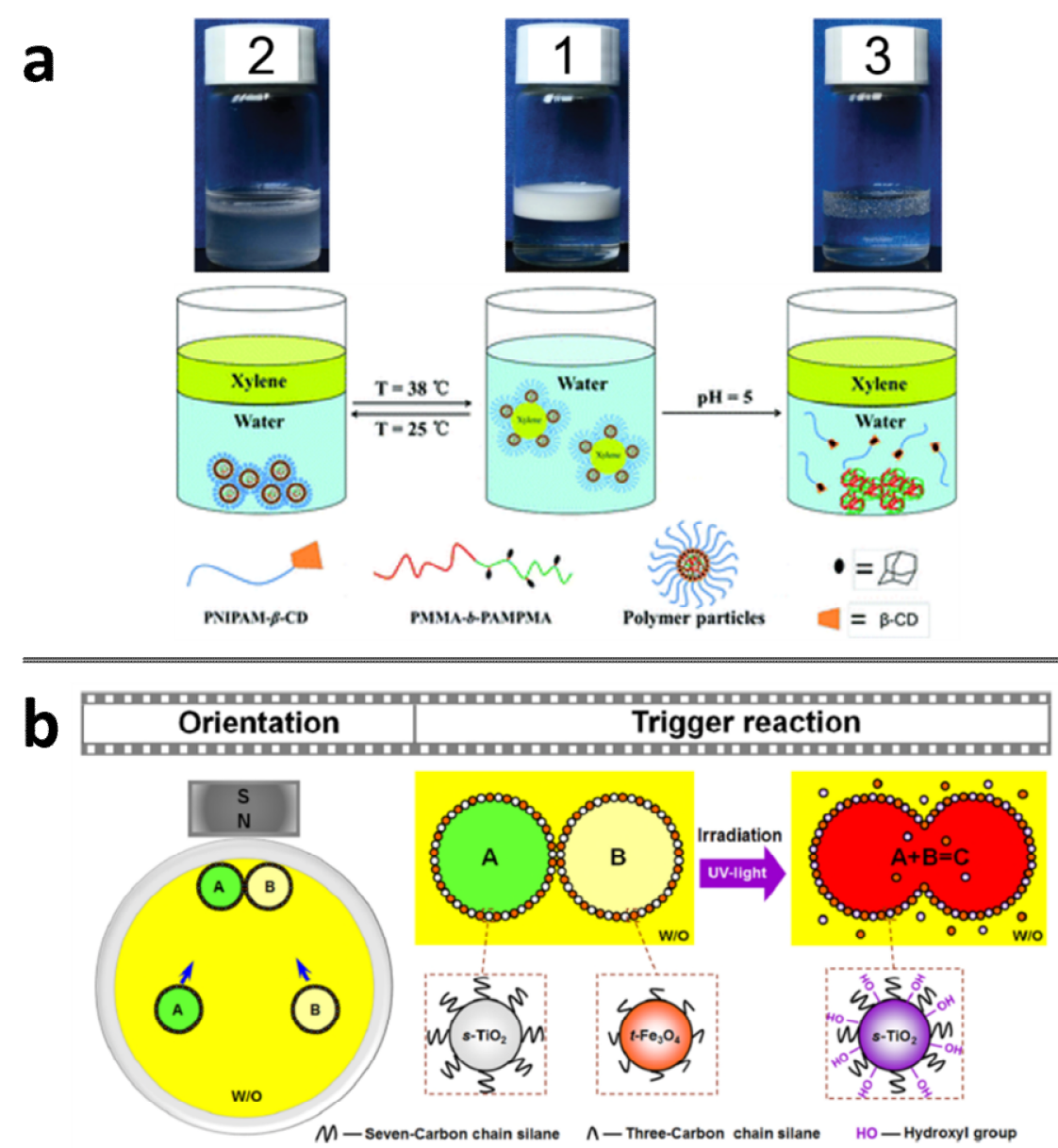

Figure 3. Examples of multi-component Pickering emulsions capable of responsiveness to multiple stimuli. a) Top panel shows photographs of an amphiphilic double dynamer stabilised Pickering emulsion (1), a broken emulsion upon thermal treatment (2) and a broken emulsion upon $\mathrm{pH}$ treatment (3). Photographs correspond to the behaviour shown in the schematic in the bottom panel; images adapted, with permission, from the Royal Society of Chemistry copyright 2018.[104] b) Schematic showing dual magnetic and UV responsive Pickering emulsions stabilised by magnetic and titania particles. In the left panel, orientation of stable emulsion droplets occurs alongside a magnet. In the right panels, UV-triggered coalescence and chemical reaction can occur; image adapted, with permission from the American Chemical Society copyright 2017.[109]

\section{Conclusions and Future Directions}

Since their first description by Pickering and Ramsden over a century ago, particle stabilised emulsions have been widely investigated for a variety of applications. Their improved biocompatibility, stability and ease of modification/tunability based on the chosen particle design, make them a favourable alternative to conventional surfactant-stabilised emulsions. Whilst Pickering emulsions are widely used in the food and cosmetics industry, advances in material design have not been widely translated to clinical applications such as biosensing and therapeutic delivery. The use of Pickering emulsions for biodelivery has been probed in the form of topical, oral and parenteral delivery formulations relying on diffusion-based therapeutic release (Figure 4a). Future research directions of this broad topic are already beginning to emerge. Recently, there have been efforts to utilise naturally occurring biodegradable and biocompatible materials. Li et al., for example, have developed Pickering 
emulsions using combinations of corn prolamine zein and polysaccharide gum Arabic to encapsulate thymol, a natural anti-microbial agent.[111] This approach may overcome issues associated with poor biocompatibility of stabilising particles, and biodegradation may potentially facilitate sustained cargo release.

The development of stimuli-responsive Pickering emulsions is a rapidly growing field, thanks to their potential for targeted delivery directly at a site of disease and the possibility of remote control, either by a trained clinician, or through exploiting a disease's own pathology and microenvironment to trigger therapeutic delivery. This level of control could improve therapeutic efficacy and reduce side effects through decreased systemic exposure, of massive importance to the safety and well-being of patients. Stimuli-responsive Pickering emulsions can be categorised based on their sensitivity to external environments such as $\mathrm{pH}$, light, temperature, magnetic fields or (bio)chemical entities, such as disease pathology-specific enzymes, proteins or acidity (Figure 4b, Tables 1 and 2). The pre-existence of clinical hardware, such as NIR for photodynamic therapy, which could be used to trigger lightstimulated Pickering emulsions, for example, could make these systems even more attractive to the pharmaceutical and clinical industry.

Further enhancement of the idea of triggered-delivery is more recently moving towards Pickering emulsions which are sensitive to a number of stimuli. The future of this area, we believe, lies in multi-component stabilising particle emulsion systems, wherein a number of different emulsifiers, each with their own independent response to a stimulus, are exploited. These would enable either staggered release from multi-shelled Pickering emulsions, or else release only at a site with the 'perfect' cocktail of stimuli. Whilst the benefits of such preciserelease systems are clear, current research needs to push further to explore the full potential of these multi-responsive dynamic systems, in particular towards in vitro and in vivo behaviour. It is clear, therefore that the area of Pickering emulsions for biomedical applications has enormous potential, with a lot still to be achieved, and we should see continuing and increased activity in coming years.

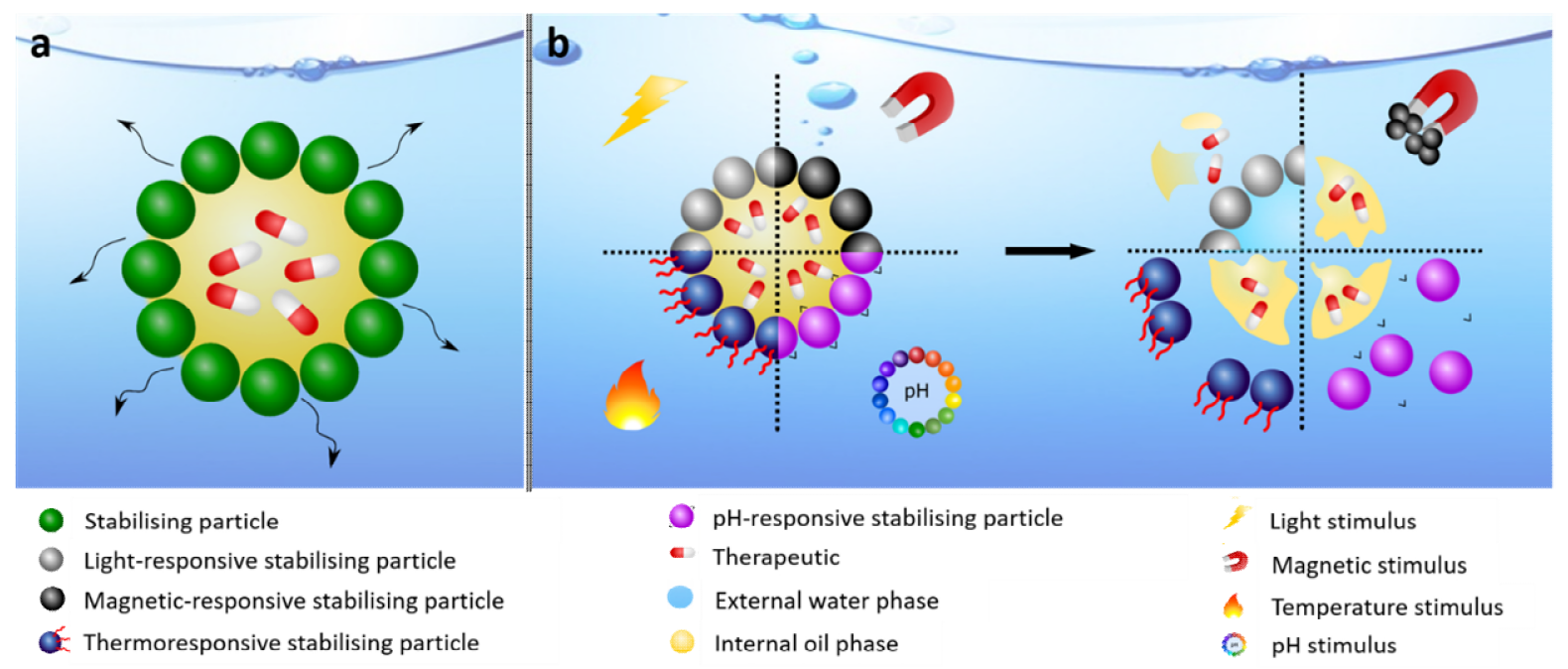

Figure 4. a) Schematic showing passive diffusion-controlled release of cargo from a Pickering emulsion. b) Schematic showing actuated release of cargo from stimuli-responsive Pickering emulsions. Clockwise: exposure to a magnetic field results in removal of magnetic nanoparticles and demulsification; alteration of $\mathrm{pH}$ causes a change of surface charge and wettability, destabilising the emulsions; temperature dependent conformation changes of 
polymer-based Pickering emulsions result in demulsification; and irradiation of light can lead to phase inversion of the Pickering emulsion.

\section{Acknowledgements}

The authors acknowledge financial support from the EPSRC through the Centre for Doctoral Training in Advanced Therapeutics and Nanomedicines (EP/L01646X, supporting CLGH), and the Centre for Doctoral Training in Molecular Modelling and Materials Science (EP/L015862/1, supporting MAP) in support of BASF SE.

\section{Declaration of Interest}

None.

\section{References}

[1] Ramsden W. Separation of solids in the surface-layers of solutions and "suspensions" (observations on surface-membranes, bubbles, emulsions, and mechanical coagulation). Preliminary Account. Proc R Soc London 1903;72:156-64. doi:10.1098/rspl.1903.0034.

[2] Pickering SU. CXCVI._Emulsions. J Chem Soc, Trans 1907;91:2001-21. doi:10.1039/CT9079102001.

[3] Binks BP. Particles as surfactants - similarities and differences. Curr Opin Colloid Interface Sci 2002;7:21-41. doi:10.2118/141256-PA.

[4] Chevalier $Y$, Bolzinger MA. Emulsions stabilized with solid nanoparticles: Pickering emulsions. Colloids Surfaces A Physicochem Eng Asp 2013;439:23-34. doi:10.1016/j.colsurfa.2013.02.054.

[5] Averyard R, Binks BP, Clint JH. Emulsions stabilised solely by colloidal particles. Adv Colloid Interface Sci 2003;100-102:503-46. doi:10.1007/978-3-642-04417-5_2.

[6] Tang J, Quinlan PJ, Tam KC. Stimuli-responsive Pickering emulsions: Recent advances and potential applications. Soft Matter 2015;11:3512-29. doi:10.1039/c5sm00247h.

[7] Wu J, Ma GH. Recent studies of Pickering emulsions: Particles make the difference. Small 2016;12:4633-48. doi:10.1002/smll.201600877.

[8] Marto J, Ascenso A, Simoes S, Almeida AJ, Ribeiro HM. Pickering emulsions: challenges and opportunities in topical delivery. Expert Opin Drug Deliv 2016;13:1093107. doi:10.1080/17425247.2016.1182489.

*[9] Chen J, Zhu C, Yang Z, Wang P, Yue Y, Kitaoka T. Thermally tunable Pickering emulsions stabilized by carbon-dot-incorporated core-shell nanospheres with fluorescence "on-off" behavior. Langmuir 2018;34:273-83. doi:10.1021/acs.langmuir.7b03490.

Using carbon dots incorporated into pNIPAM and grafted onto cellulose acetate nanocrystals, the authors demonstrated that the conformational transition termperature of pNIPAM can be used to control the fluorescence of the carbon dots. Such systems offer potential in sensing and imaging applications.

[10] Frelichowska J, Bolzinger MA, Valour JP, Mouaziz H, Pelletier J, Chevalier Y. Pickering w/o emulsions: Drug release and topical delivery. Int J Pharm 2009;368:7-15. doi:10.1016/j.ijpharm.2008.09.057.

[11] Frelichowska J, Bolzinger MA, Pelletier J, Valour JP, Chevalier Y. Topical delivery of lipophilic drugs from o/w Pickering emulsions. Int J Pharm 2009;371:56-63. doi:10.1016/j.ijpharm.2008.12.017.

*[12] Salerno A, Bolzinger MA, Rolland P, Chevalier Y, Josse D, Briançon S. Pickering emulsions for skin decontamination. Toxicol Vitr 2016;34:45-54. doi:10.1016/j.tiv.2016.03.005.

In the first example of Pickering emulsions for skin decontamination, the authors 
herein have developed silica based Pickering emulsions combined with a scavenging agent for the chemical warfare agent VX. The formulation was demonstrated to have enhanced scavenging properties when compared with the aqueous scavenging agent alone, with excellent efficiency towards new types of skin decontamination systems.

[13] Elmotasem H, Farag HK, Salama AAA. In vitro and in vivo evaluation of and oral sustained release hepatoprotective caffeine loaded w/o Pickering emulsion formula Containing wheat germ oil and stabilized by magnesium oxide nanoparticles. Int $\mathrm{J}$ Pharm 2018;547:83-96. doi:10.1016/j.jpharm.2018.05.038.

[14] Laredj-Bourezg F, Bolzinger MA, Pelletier J, Chevalier Y. Pickering emulsions stabilized by biodegradable block copolymer micelles for controlled topical drug delivery. Int J Pharm 2017;531:134-42. doi:10.1016/j.ijpharm.2017.08.065.

[15] Simeone DM. Pancreatic cancer stem cells: Implications for the treatment of pancreatic cancer. Clin Cancer Res 2008;14:5646-8. doi:10.1158/1078-0432.CCR-08-0584.

[16] Asfour MH, Elmotasem H, Mostafa DM, Salama AAA. Chitosan based Pickering emulsion as a promising approach for topical application of rutin in a solubilized form intended for wound healing: In vitro and in vivo study. Int J Pharm 2017;534:325-38. doi:10.1016/j.ijpharm.2017.10.044.

[17] Cossu A, Wang MS, Chaudhari A, Nitin N. Antifungal activity against Candida albicans of starch Pickering emulsion with thymol or amphotericin B in suspension and calcium alginate films. Int J Pharm 2015;493:233-42. doi:10.1016/j.jjpharm.2015.07.065.

[18] Hu JW, Yen MW, Wang AJ, Chu IM. Effect of oil structure on cyclodextrin-based Pickering emulsions for bupivacaine topical application. Colloids Surfaces B Biointerfaces 2018;161:51-8. doi:10.1016/j.colsurfb.2017.10.001.

[19] Leclercq L, Nardello-Rataj V. Pickering emulsions based on cyclodextrins: A smart solution for antifungal azole derivatives topical delivery. Eur J Pharm Sci 2016;82:12637. doi:10.1016/j.ejps.2015.11.017.

[20] Wang Q, Hu C, Zoghbi A, Huang J, Xia Q. Oil-in-oil-in-water pre-double emulsions stabilized by nonionic surfactants and silica particles: A new approach for topical application of rutin. Colloids Surfaces A Physicochem Eng Asp 2017;522:399-407. doi:10.1016/j.colsurfa.2017.02.067.

[21] Pan J, Li L, Hang H, Wu R, Dai X, Shi W, et al. Fabrication and evaluation of magnetic/hollow double-shelled imprinted sorbents formed by Pickering emulsion polymerization. Langmuir 2013;29:8170-78. doi:10.1021/la4015288.

[22] Zhang K, Wu W, Guo K, Chen J, Zhang P. Synthesis of temperature-responsive poly( $\mathrm{N}$-isopropyl acrylamide)/poly(methyl methacrylate)/silica hybrid capsules from inverse Pickering emulsion polymerization and their application in controlled drug release. Langmuir 2010;26:7971-80. doi:10.1021/la904841m.

[23] Zhu X, Zhang S, Zhang L, Liu H, Hu J. Interfacial synthesis of magnetic PMMA@ $\mathrm{Fe}_{3} \mathrm{O}_{4} / \mathrm{Cu}_{3}(\mathrm{BTC})_{2}$ hollow microspheres through one-pot Pickering emulsion and their application as drug delivery. RSC Adv 2016;6:58511-5. doi:10.1039/c6ra11077k.

[24] Hajizadeh S, Kettisen K, Gram M, Bülow L, Ye L. Composite imprinted macroporous hydrogels for haemoglobin purification from cell homogenate. J Chromatogr A 2017;1534:22-31. doi:10.1016/j.chroma.2017.12.038.

[25] Marto J, Gouveia LF, Gonçalves L, Chiari-Andréo BG, Isaac V, Pinto P, et al. Design of novel starch-based Pickering emulsions as platforms for skin photoprotection. $J$ Photochem Photobiol B Biol 2016;162:56-64. doi:10.1016/j.jphotobiol.2016.06.026.

[26] Binks BP, Fletcher PDI, Johnson AJ, Marinopoulos I, Crowther JM, Thompson MA. Evaporation of particle-stabilized emulsion sunscreen films. ACS Appl Mater Interfaces 2016;8:21201-13. doi:10.1021/acsami.6b06310.

[27] Marto J, Ascenso A, Gonçalves LM, Gouveia LF, Manteigas P, Pinto P, et al. Melatoninbased pickering emulsion for skin's photoprotection. Drug Deliv 2016;23:1594-607. doi:10.3109/10717544.2015.1128496. 
[28] Sy PM, Anton N, Idoux-Gillet Y, Dieng SM, Messaddeq N, Ennahar S, et al. Pickering nano-emulsion as a nanocarrier for $\mathrm{pH}$-triggered drug release. Int $\mathrm{J}$ Pharm 2018;549:299-305. doi:10.1016/j.ijpharm.2018.07.066.

*[29] Yi T, Liu C, Zhang J, Wang F, Wang J, Zhang J. A new drug nanocrystal self-stabilized Pickering emulsion for oral delivery7 of silybin. Eur J Pharm Sci 2017;96:420-7. doi:10.1016/j.ejps.2016.08.047.

This article is one of the first which uses a therapeutic drug (silybin) as a Pickering emulsion stabiliser. Silybin nanocrystals were used as the emulsion stabiliser, and demonstrated enhanced and faster drug-release properties than non-Pickering emulsion silybin-containing formulations. The authors demonstrated that these nanocrystalline drug self-stabilised Pickering emulsions could be promising for the oral delivery or poorly soluble drugs.

[30] Deschamps F, Harris KR, Moine L, Li W, Tselikas L, Isoardo T, et al. Pickering-emulsion for liver trans-arterial chemo-embolization with oxaliplatin. Cardiovasc Intervent Radiol 2018;41:781-8. doi:10.1007/s00270-018-1899-y.

[31] Szewczuk M, Hrynyk M, Ellis JP, Haxho F, Allison S, Steele JAM, et al. Therapeutic designed poly (lactic-co-glycolic acid) cylindrical oseltamivir phosphate-loaded implants impede tumor neovascularization, growth and metastasis in mouse model of human pancreatic carcinoma. Drug Des Devel Ther 2015:4573. doi:10.2147/DDDT.S90170.

[32] Allison Logan S, Brissenden AJ, Szewczuk MR, Neufeld RJ. Combinatorial and sequential delivery of gemcitabine and oseltamivir phosphate from implantable poly(D,L-lactic-co-glycolic acid) cylinders disables human pancreatic cancer cell survival. Drug Des Devel Ther 2017;11:2239-50. doi:10.2147/DDDT.S137934.

*[33] Wood K, Szewczuk MR, Rousseau D, Neufeld RJ. Oseltamivir phosphate released from injectable Pickering emulsions over an extended term disables human pancreatic cancer cell survival. Oncotarget 2018;9:12754-68. doi:10.18632/oncotarget.24339.

Pickering water-in-oil emulsions, where molten glycerol monostearate crystallised at the surface of micron-sized water droplets and formed protective solid shells, were investigated for the sustained release of the therapeutic oseltamivir phosphate. The authors demonstrated the sustained release of oseltamivir phosphate, over 30-days, which, interestingly, was near linear and had successful therapeutic effect of against pancreatic cancer cells.

${ }^{* *}$ [34] Xia Y, Wei J, Du Y, Wan T, Ma X, An W, et al. Exploiting the pliability and lateral mobility of Pickering emulsion for enhanced vaccination. Nat Mater 2018;17:187-94. doi:10.1038/NMAT5057.

Herein, PLGA-stabilised Pickering emulsions immobilised with antigens were developed that retain the force-dependent deformability and lateral mobility of presented antigens while displaying high biosafety and antigen-loading capabilities. Compared with solid particles and conventional surfactantstabilised emulsions, the optimised Pickering emulsions enhanced the recruitment, antigen uptake and activation of antigen-presenting cells as the first example of a new adjuvant for vaccine delivery.

[35] Huang CY, Huang CH, Liu SJ, Chen HW, Leng CH, Chong P, et al. Polysorbasome: A colloidal vesicle contoured by polymeric bioresorbable amphiphiles as an immunogenic depot for vaccine delivery. ACS Appl Mater Interfaces 2018;10:12553-61. doi:10.1021/acsami.8b03044.

[36] Blanco E, Shen H, Ferrari M. Principles of nanoparticle design for overcoming biological barriers to drug delivery. Nat Biotechnol 2015;33:941-51. doi:10.1038/nbt.3330.

[37] Decuzzi P, Godin B, Tanaka T, Lee SY, Chiappini C, Liu X, et al. Size and shape effects in the biodistribution of intravascularly injected particles. J Control Release 2010;141:320-7. doi:10.1016/j.jconrel.2009.10.014.

[38] Shen X, Ye L. Molecular imprinting in Pickering emulsions: A new insight into molecular recognition in water. Chem Commun 2011;47:10359-61. doi:10.1039/c1cc13899e.

[39] Haupt K, Mosbach K. Molecularly imprinted polymers and their use in biomimetic 
sensors. Chem Rev 2000;100:2495-504. doi:10.1021/cr990099w.

[40] Kujawska M, Zhou T, Trochimczuk AW, Ye L. Synthesis of naproxen-imprinted polymer using Pickering emulsion polymerization. $J$ Mol Recognit 2018;31:1-12. doi:10.1002/jmr.2626.

[41] Shen X, Zhou T, Ye L. Molecular imprinting of protein in Pickering emulsion. Chem Commun 2012;48:8198-200. doi:10.1039/c2cc33572g.

[42] Zhou T, Shen X, Chaudhary S, Ye L. Molecularly imprinted polymer beads prepared by pickering emulsion polymerization for steroid recognition. J Appl Polym Sci 2014;131:17. doi:10.1002/app.39606.

[43] Shen X, Svensson Bonde J, Kamra T, Bülow L, Leo JC, Linke D, et al. Bacterial imprinting at pickering emulsion interfaces. Angew Chemie - Int Ed 2014;53:10687-90. doi:10.1002/anie.201406049.

[44] Yang J, Li Y, Wang J, Sun X, Cao R, Sun H, et al. Molecularly imprinted polymer microspheres prepared by Pickering emulsion polymerization for selective solid-phase extraction of eight bisphenols from human urine samples. Anal Chim Acta 2015;872:35-45. doi:10.1016/j.aca.2015.02.058.

[45] Staats HF, Burkhart DJ. Softness makes it better. Nat Publ Gr 2018;17:113-4. doi:10.1038/nmat5072.

[46] Tang Q, Yu B, Gao L, Cong H, Song N, Lu C. Stimuli responsive nanoparticles for controlled anti-cancer drug release. Curr Med Chem 2018;25:1837-66. doi:10.2174/0929867325666180111095913.

[47] Breckwoldt MO, Chen JW, Stangenberg L, Aikawa E, Rodriguez E, Qiu S, et al. Tracking the inflammatory response in stroke in vivo by sensing the enzyme myeloperoxidase. Proc Natl Acad Sci 2008;105:18584-9. doi:10.1073/pnas.0803945105.

[48] Noy D, Solomonov I, Sinkevich O, Arad T, Kjaer K, Sagi I. Zinc-amyloid $\beta$ interactions on a millisecond time-scale stabilize non-fibrillar Alzheimer-related species. J Am Chem Soc 2008;130:1376-83. doi:10.1021/ja076282l.

[49] Gillies RJ, Raghunand N, Garcia-Martin ML, Gatenby RA. pH imaging. IEEE Eng Med Biol Mag 2004;23:57-64. doi:10.1109/MEMB.2004.1360409.

[50] Davies GL, Kramberger I, Davis JJ. Environmentally responsive MRI contrast agents. Chem Commun 2013;49:9704-21. doi:10.1039/c3cc44268c.

*[51] Guo H, Wang Y, Huang Y, Huang F, Li S, Shen Y, et al. A GO@PLA@HA composite microcapsule: Its preparation and multistage and controlled drug release. Eur J Inorg Chem 2017;2017:3312-21. doi:10.1002/ejic.201700193.

A new type of double Pickering emulsion multiple drug-release system, graphene oxide (GO)@polylactic acid (PLA)@hydroxyapatite (HA) composite microcapsule, was constructed by the authors. The controlled release of 3 different hosted drugs was realised by the degradation of HA in acidic environments, illustrating the pH-triggered drug-release behaviour of HA. Further, step-wise sustained release of the 3 drugs was observed as a result of the double Pickering emulsion design.

${ }^{*}$ [52] Bai RX, Xue LH, Dou RK, Meng SX, Xie CY, Zhang Q, et al. Light-triggered release from Pickering emulsions stabilized by $\mathrm{TiO}_{2}$ nanoparticles with tailored wettability. Langmuir 2016;32:9254-64. doi:10.1021/acs.langmuir.6b02329.

In this work, the authors described a new strategy for light-triggered Pickering emulsions as smart soft vehicles for on-demand release. UV-induced controlled release of a cargo occurred by demulsification through changes in the wettability of $\mathrm{TiO}_{2}$ nanoparticles. Pickering emulsions sensitive to visible light were also established based on nitrogen doping of $\mathrm{TiO}_{2}$ nanoparticle emulsifiers, making this a broadly applicable triggered release system.

**[53] Hong C, Park JH, Lee S, Rhoo KY, Lee JT, Paik SR. Fabrication of protease-sensitive and light-responsive microcapsules encompassed with single layer of gold nanoparticles by using self-assembly protein of $\alpha$-synuclein. ACS Appl Mater Interfaces 
2018;10:26628-40. doi:10.1021/acsami.8b07661.

Using gold nanoparticles coated with $\alpha$-synuclein as emulsifiers, the authors developed a multifunctional system that underwent controlled cargo release upon exposure to protease; the system also exhibited light-sensitivity and photothermal properties. Such systems could hold exceptional promise as targeted cargo-carriers capable of precision pathological site recognition, of use for future sensor and therapeutic applications.

[54] Whitby CP, Fischer FE, Fornasiero D, Ralston J. Shear-induced coalescence of oil-inwater Pickering emulsions. J Colloid Interface Sci 2011;361:170-7. doi:10.1016/j.jcis.2011.05.046.

[55] Chen G, Tan P, Chen S, Huang J, Wen W, Xu L. Coalescence of pickering emulsion droplets induced by an electric field. Phys Rev Lett 2013;110:1-5. doi:10.1103/PhysRevLett.110.064502.

[56] Zhou Q, Sun Y, Yi S, Wang K, Luo G. Investigation of droplet coalescence in nanoparticle suspensions by a microfluidic collision experiment. Soft Matter 2016;12:1674-82. doi:10.1039/c5sm02924d.

[57] Hou L, Ren Y, Jia Y, Deng X, Liu W, Feng X, et al. Continuously electrotriggered core coalescence of double-emulsion drops for microreactions. ACS Appl Mater Interfaces 2017;9:12282-9. doi:10.1021/acsami.7b00670.

[58] Yang Y, Fang Z, Chen X, Zhang W, Xie Y, Chen Y, et al. An overview of pickering emulsions: Solid-particle materials, classification, morphology, and applications. Front Pharmacol 2017;8:1-20. doi:10.3389/fphar.2017.00287.

[59] Wike-Hooley JL, Haveman J, Reinhold HS. The relevance of tumour $\mathrm{pH}$ to the treatment of malignant disease. Radiother Oncol 1984;2:343-66. doi:10.1007/978-3-658-098377.

[60] Anjali TG, Basavaraj MG. General destabilization mechanism of pH-responsive Pickering emulsions. Phys Chem Chem Phys 2017:16-9. doi:10.1039/c7cp04665k.

[61] Li J, Stover HDH. Doubly pH-Responsive Pickering Emulsion. Langmuir 2008:1323740. doi:10.1021/la802619m.

[62] Haase MF, Grigoriev D, Moehwald H, Tiersch B, Shchukin DG. Encapsulation of amphoteric substances in a pH-sensitive Pickering emulsion. J Phys Chem C 2010:17304-10. doi: 10.1021/jp104052s.

[63] Liu K, Jiang J, Cui Z, Binks BP. pH-responsive Pickering emulsions stabilized by silica nanoparticles in combination with a conventional zwitterionic surfactant. Langmuir 2017:33:2296-2305. doi:10.1021/acs.langmuir.6b04459.

*[64] Liu M, Chen X, Yang Z, Xu Z, Hong L, Ngai T. Tunable Pickering emulsions with environmentally responsive hairy silica nanoparticles. ACS Appl Mater Interfaces 2016;8:32250-8. doi:10.1021/acsami.6b11931.

"Hairy" silica nanoparticles surface coated with mixed polymer brush-coated nanoparticles were produced as Pickering emulsion stabilisers. These "hairy" nanoparticles can facilitate the production of $\mathrm{O} / \mathrm{W} / \mathrm{O}$ emulsions that are not attainable from conventional Pickering emulsifiers. The functionalised hairy silica nanoparticles can be further linked together utilising poly(acrylic acid) as a reversible linker to form supramolecular colloidosomes, which show $\mathrm{pH}$ dependent release of cargo.

[65] Motornov M, Sheparovych R, Lupitskyy R, MacWilliams E, Hoy O, Luzinov I, et al. Stimuli-responsive colloidal systems from mixed brush-coated nanoparticles. Adv Funct Mater 2007;17:2307-14. doi:10.1002/adfm.200600934.

[66] Ma C, Bi X, Ngai T, Guangzhao Z. Polyurethane-based nanoparticles as stabilizers for oil-in-water or water-in-oil Pickering emulsions. J Mater Chem A, 2013;1:5353-60. doi:10.1039/c3ta00123g.

[67] Tu F, Lee D. Shape-changing and amphiphilicity-reversing Janus particles with $\mathrm{pH}$ responsive surfactant properties. J Am Chem Soc 2014;136:9999-10006. doi:10.1021/ja503189r.

[68] Liu H, Wang C, Zou S, Wei Z, Tong Z. Simple, reversible emulsion system switched by 
$\mathrm{pH}$ on the basis of chitosan without any hydrophobic modification. Langmuir 2012;28:11017-24. doi:10.1021/la3021113.

[69] Pang K, Ding B, Liu X, Wu H, Duan Y, Zhang J. High-yield preparation of a zwitterionically charged chitin nanofiber and its application in a doubly $\mathrm{pH}$-responsive Pickering emulsion. Green Chem 2017;19:3665-70. doi:10.1039/c7gc01592e.

[70] Ruan Q, Guo J, Wan Z, Ren J, Yang X. pH switchable Pickering emulsion based on soy peptides functionalized calcium phosphate particles. Food Hydrocoll 2017;70:21928. doi:10.1016/j.foodhyd.2017.03.023.

[71] Chen N, Dempere LA, Tong Z. Synthesis of pH-Responsive lignin-based nanocapsules for controlled release of hydrophobic molecules. ACS Sustain Chem Eng 2016. doi:10.1021/acssuschemeng.6b01209.

[72] Zhang W, Sun X, Fan X, Li M, He G. Pickering emulsions stabilized by hydrophobically modified alginate nanoparticles: Preparation and $\mathrm{pH}$-responsive performance in vitro. J. Dispers. Sci. Technol., vol. 39, Taylor \& Francis; 2018, p. 367-74. doi:10.1080/01932691.2017.1320223.

[73] Nan F, Wu J, Qi F, Liu Y, Ngai T, Ma G. Uniform chitosan-coated alginate particles as emulsifiers for preparation of stable Pickering emulsions with stimulus dependence. Colloids Surfaces A Physicochem Eng Asp 2014;456:246-52. doi:10.1016/j.colsurfa.2014.05.017.

[74] Zhu Y, Wang J, Li X, Zhao D, Sun J, Liu X. Self-assembly and emulsification of dopamine-modified hyaluronan. Carbohydr Polym 2015;123:72-9. doi:10.1016/j.carbpol.2015.01.030.

[75] Rwei AY, Wang W, Kohane DS. Photoresponsive nanoparticles for drug delivery. Nano Today 2015;10:451-67. doi:10.1016/j.nantod.2015.06.004.

[76] Huang X, El-Sayed IH, Qian W, El-Sayed MA. Cancer cell imaging and photothermal therapy in the near-infrared region by using gold nanorods. J Am Chem Soc 2006;128:2115-20. doi:10.1021/ja057254a.

[77] Wan MT, Lin JY. Current evidence and applications of photodynamic therapy in dermatology. Clin Cosmet Investig Dermatol 2014;7:145-63.

[78] Wu Y, Zhang C, Qu X, Liu Z, Yang Z. Light-triggered reversible phase transfer of composite colloids. Langmuir 2010;26:9442-8. doi:10.1021/la100458j.

[79] Chen Z, Zhou L, Bing W, Zhang Z, Li Z, Ren J, et al. Light controlled reversible inversion of nanophosphor-stabilized Pickering emulsions for biphasic enantioselective biocatalysis. J Am Chem Soc 2014:136:7498-7504. doi:10.1021/ja503123m.

[80] Chen Y, Li Z, Wang H, Pei Y, Shi Y, Wang J. Visible light-controlled inversion of Pickering emulsions stabilized by functional silica microspheres. Langmuir 2018:34:2784-90. doi:10.1021/acs.langmuir.7b03822.

[81] Zhang Q, Bai RX, Guo T, Meng T. Switchable Pickering emulsions stabilized by awakened $\mathrm{TiO}_{2}$ nanoparticle emulsifiers using UV/dark actuation. ACS Appl Mater Interfaces 2015;7:18240-6. doi:10.1021/acsami.5b06808.

[82] Armstrong BK, Kricker A. The epidemiology of UV induced skin cancer. J Photochem Photobiol B Biol 2001;63:8-18. doi:http://dx.doi.org/10.1159/000343182.

[83] Ekkelenkamp AE, Elzes MR, Engbersen JFJ, Paulusse JMJ. Responsive crosslinked polymer nanogels for imaging and therapeutics delivery. J Mater Chem B 2018;6:21035. doi:10.1039/c7tb02239e.

[84] Wang X, Zeng M, Yu YH, Wang H, Mannan MS, Cheng Z. Thermosensitive ZrPPNIPAM Pickering Emulsifier and the Controlled-Release Behavior. ACS Appl Mater Interfaces 2017;9:7852-8. doi:10.1021/acsami.6b16690.

[85] Tsuji S, Kawaguchi $\mathrm{H}$. Thermosensitive pickering emulsion stabilized by poly $(\mathrm{N}-$ isopropylacrylamide)-carrying particles. Langmuir 2008;24:3300-5. doi:10.1021/la701780g.

[86] Zoppe JO, Venditti RA, Rojas OJ. Pickering emulsions stabilized by cellulose nanocrystals grafted with thermo-responsive polymer brushes. J Colloid Interface Sci 2012;369:202-9. doi:10.1016/j.jcis.2011.12.011.

[87] Gao Q, Wang C, Liu H, Wang C, Liu X, Tong Z. Suspension polymerization based on 
inverse Pickering emulsion droplets for thermo-sensitive hybrid microcapsules with tunable supracolloidal structures. Polymer (Guildf) 2009;50:2587-94. doi:10.1016/j.polymer.2009.03.049.

[88] Yang H, Liang F, Wang X, Chen Y, Zhang C, Wang Q, et al. Responsive Janus composite nanosheets. Macromolecules 2013;46:2754-9. doi:10.1021/ma400261y.

[89] Zhu Y, Fu T, Liu K, Lin Q, Pei X, Jiang J, et al. Thermoresponsive Pickering emulsions stabilized by silica nanoparticles in combination with alkyl polyoxyethylene ether nonionic surfactant. Langmuir 2017:33:5724-33. doi:10.1021/acs.langmuir.7b00273.

[90] Ternent L, Mayoh DA, Lees MR, Davies GL. Heparin-stabilised iron oxide for MR applications: A relaxometric study. J Mater Chem B 2016;4:3065-74. doi:10.1039/c6tb00832a.

[91] Berry CC. Progress in functionalization of magnetic nanoparticles for applications in biomedicine. J Phys D Appl Phys 2009;42. doi:10.1088/0022-3727/42/22/224003.

[92] Peng J, Liu Q, Xu Z, Masliyah J. Synthesis of interfacially active and magnetically responsive nanoparticles for multiphase separation applications. Adv Funct Mater 2012;22:1732-40. doi:10.1002/adfm.201102156.

[93] Zhang K, Wu W, Guo K, Chen JF, Zhang PY. Magnetic polymer enhanced hybrid capsules prepared from a novel Pickering emulsion polymerization and their application in controlled drug release. Colloids Surfaces A Physicochem Eng Asp 2009;349:1106. doi:10.1016/j.colsurfa.2009.08.005.

[94] Lin KYA, Yang $\mathrm{H}$, Petit $\mathrm{C}$, Lee W der. Magnetically controllable Pickering emulsion prepared by a reduced graphene oxide-iron oxide composite. J Colloid Interface Sci 2015;438:296-305. doi:10.1016/j.jcis.2014.10.015.

[95] Kaiser A, Liu T, Richtering W, Schmidt AM. Magnetic capsules and pickering emulsions stabilized by core-shell particles. Langmuir 2009;25:7335-41. doi:10.1021/la900401f.

[96] Qiao X, Zhou J, Binks BP, Gong X, Sun K. Magnetorheological behavior of Pickering emulsions stabilized by surface-modified $\mathrm{Fe}_{3} \mathrm{O}_{4}$ nanoparticles. Colloids Surfaces $\mathrm{A}$ Physicochem Eng Asp 2012;412:20-8. doi:10.1016/j.colsurfa.2012.06.026.

[97] Melle S, Lask M, Fuller GG. Pickering emulsions with controllable stability. Langmuir 2005;21:2158-62. doi:10.1021/la047691n.

[98] Xiao W, Zeng X, Lin H, Han K, Jia HZ, Zhang XZ. Dual stimuli-responsive multi-drug delivery system for the individually controlled release of anti-cancer drugs. Chem Commun 2015;51:1475-8. doi:10.1039/c4cc08831j.

[99] Cao Y, Wang Z, Zhang S, Wang Y. Synergetic regulation of $\mathrm{CO}_{2}$ and light for controllable inversion of Pickering emulsions. Mater Chem Front 2017;1:2136-42. doi:10.1039/C7QM00275K.

[100] Wang F, Yu X, Yang Z, Duan H, Zhang Z, Liu H. Dual pH-and light-responsive amphiphilic random copolymer nanomicelles as particulate emulsifiers to stabilize the oil/water interface. J Phys Chem C 2018;122:18995-9003. doi:10.1021/acs.jpcc.8b05065.

[101] Low LE, Tey BT, Ong BH, Tang SY. Unravelling $\mathrm{pH}$-responsive behaviour of $\mathrm{Fe}_{3} \mathrm{O}_{4} @ \mathrm{CNCs}$-stabilized Pickering emulsions under the influence of magnetic field. Polym (United Kingdom) 2018;141:93-101. doi:10.1016/j.polymer.2018.03.001.

[102] Zhang Y, Guo S, Ren X, Liu X, Fang Y. $\mathrm{CO}_{2}$ and redox dual responsive Pickering emulsion. Langmuir 2017;33:12973-81. doi:10.1021/acs.langmuir.7b02976.

[103] Chen Q, Xu Y, Cao X, Qin L, An Z. Core cross-linked star (CCS) polymers with temperature and salt dual responsiveness: Synthesis, formation of high internal phase emulsions (HIPEs) and triggered demulsification. Polym Chem 2014;5:175-85. doi:10.1039/c3py00942d.

[104] Zeng T, Yang D, Li H, Cheng C, Gao Y. The fabrication of amphiphilic double dynamers for responsive Pickering emulsifiers. Polym Chem 2018;9:627-36. doi:10.1039/c7py02067h.

[105] Tang J, Lee MFX, Zhang W, Zhao B, Berry RM, Tam KC. Dual responsive Pickering emulsion stabilized by poly[2-(dimethylamino) ethyl methacrylate] grafted cellulose nanocrystals. Biomacromolecules 2014;15:3052-60. doi:10.1021/bm500663w. 
[106] Zhou G, Zhao Y, Hu J, Shen L, Liu W, Yang X. A new drug-loading technique with high efficaciency and sustained-releasing ability via the Pickering emulsion interfacial assembly of temperature/pH- sensitive nanogels. React Funct Polym 2013;73:153743. doi:10.1016/j.reactfunctpolym.2013.08.004.

[107] Low LE, Tey BT, Ong BH, Chan ES, Tang SY. Palm olein-in-water Pickering emulsion stabilized by $\mathrm{Fe}_{3} \mathrm{O}_{4}$-cellulose nanocrystal nanocomposites and their responses to $\mathrm{pH}$. Carbohydr Polym 2017;155:391-9. doi:10.1016/j.carbpol.2016.08.091.

[108] Raju RR, Liebig F, Klemke B, Koetz J. pH-responsive magnetic Pickering Janus emulsions. Colloid Polym Sci 2018;296:1039-46. doi:10.1007/s00396-018-4321-z.

${ }^{* *}$ [109] Xie CY, Meng SX, Xue LH, Bai RX, Yang X, Wang Y, et al. Light and Magnetic DualResponsive Pickering Emulsion Micro-Reactors. Langmuir 2017;33:14139-48. doi:10.1021/acs.langmuir.7b03642.

Herein, the authors described a new type of light and magnetic dual-responsive Pickering emulsion microreactor by co-adsorption of light-sensitive titania $\left(\mathrm{TiO}_{2}\right)$ and superparamagnetic iron oxide $\left(\mathrm{Fe}_{3} \mathrm{O}_{4}\right)$ nanoparticles at the oil-water interface of emulsion droplets. In a novel advancement of triggered Pickering emulsion destabilisation, the authors use, for the first time, an external magnetic field to aggregate the microcapsules. A chemical reaction was then stimulated upon UV illumination due to the contact of the isolated reactants as a result of droplet coalescence.

[110] Brugger B, Richtering W. Magnetic, thermosensitive microgels as stimuli-responsive emulsifiers allowing for remote control of separability and stability of oil in wateremulsions. Adv Mater 2007;19:2973-8. doi:10.1002/adma.200700487.

[111] Li J, Xu X, Chen Z, Wang T, Lu Z, Hu W, et al. Zein/gum Arabic nanoparticle-stabilized Pickering emulsion with thymol as an antibacterial delivery system. Carbohydr Polym 2018;200:416-26. doi:10.1016/j.carbpol.2018.08.025. 